\title{
LONG-TERM VISUAL OUTCOME FOLLOWING ORBITAL DECOMPRESSION FOR DYSTHYROID EYE DISEASE
}

\author{
B. M. HUTCHISON and P. M. KYLE \\ Glasgow
}

\begin{abstract}
SUMMARY
The records of 33 patients who had undergone inferior and medial wall orbital decompressions for compressive optic neuropathy due to dysthyroid eye disease were reviewed. The indication for surgery in 32 patients was a reduction in Snellen visual acuity. The remaining patient had bilateral optic disc swelling but normal visual acuity. Twenty-nine patients $(88 \%)$ were treated with systemic steroids pre-operatively, which resulted in an improvement in vision in all cases. In the immediate post-operative period visual acuity either improved or the steroid-induced visual improvement was maintained as the steroids were tailed off. Long-term visual outcome, however, varied. In 19 patients $(58 \%)$ visual acuity was maintained with no requirement for additional treatment. In 12 patients $(36 \%)$ there was a subsequent deterioration in vision which responded to additional treatment with either systemic steroids, orbital radiotherapy or further orbital surgery. In 2 patients $(6 \%)$ vision continued to deteriorate despite further treatment. We conclude that although orbital decompression has resulted in the long-term preservation of visual acuity in $94 \%$ of our patients, there remains a small subgroup $(6 \%)$ in whom visual function continues to deteriorate despite all forms of treatment.
\end{abstract}

Compressive optic neuropathy is a potentially blinding condition which fortunately affects only a small proportion of patients with dysthyroid eye disease. ${ }^{1}$

Many authors have reported the benefits of surgical orbital decompression in relieving the effects of compressive optic neuropathy. ${ }^{2-7}$ The purpose of our study was to assess the long-term effect of this treatment on visual function in patients

From: Department of Ophthalmology, Southern General Hospital, Glasgow G51 4TF, UK.

Correspondence to: Dr Bruce M. Hutchison, Department of Ophthalmology, Southern General NHS Trust, 1345 Govan Road, Glasgow GS1 4TF, UK. treated in our department and to identify the proportion of patients requiring supplementary treatment.

\section{SUBJECTS AND METHODS}

The case records of 33 patients who had undergone surgical orbital decompressions for compressive optic neuropathy, as a result of dysthyroid eye disease, were reviewed. All but one patient underwent bilateral procedures, the exception being a patient who was uniocular following trauma. Twenty-eight patients were female and 5 were male. The age range was 38-77 years, mean age 56 years. Inferior and medial wall orbital decompressions were performed by the same surgeon via a translid approach. Followup interval ranged from 24 to 90 months, with a mean of 46 months.

The indication for surgery in 32 cases was a reduction in Snellen visual acuity in at least one eye. This ranged from $6 / 9$ to counting fingers (Fig. 1). The remaining patient had bilateral optic disc swelling but was asymptomatic with a visual acuity of $6 / 6$ in each eye. In addition, decreased colour vision was present in 14 patients ( $42 \%)$, visual field defects were present in 13 patients (39\%), optic disc swelling was present in 10 patients $(30 \%)$ and pupillary defects were

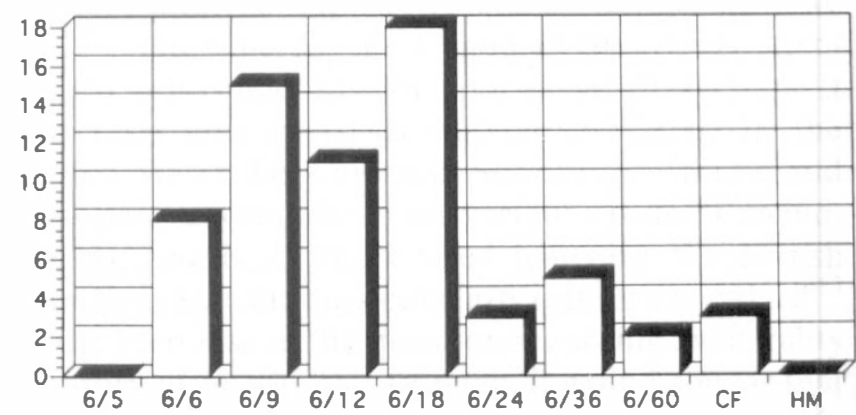

Fig. 1. Visual acuity on presentation, prior to commencement of systemic corticosteroids $(\mathrm{n}=65$ eyes $)$. 
present in 5 patients $(15 \%)$. Visual field defects were variable, with arcuate and centrocaecal scotomas being noted as well as generalised field constriction.

If only one eye was affected by compressive optic neuropathy, the fellow eye was also decompressed as a protective measure.

Patients requiring supplementary radiotherapy received 20 Gy in 10 fractions.

Orbital decompressions were also performed in our department for severe corneal exposure and cosmetic reasons. These patients were excluded from this study.

\section{RESULTS}

Pre-operatively 29 patients ( $88 \%$ ) had been started on systemic prednisolone. The initial dose was 80-120 mg daily. In all cases this resulted in an improvement in visual acuity and in most cases this treatment had been started by the referring unit. Attempts to reduce the dose of steroid commonly resulted in a deterioration in visual acuity. Several patients had begun to experience side effects of steroid therapy by the time of referral. At the time of surgery, 6 patients had been on systemic prednisolone for longer than 2 months and 18 patients were on a dose greater than $20 \mathrm{mg}$ daily. No patients had been treated by orbital radiotherapy prior to surgery.

Post-operatively, the steroid dose was gradually reduced. Mean duration of post-operative steroid treatment was 8 months. In the immediate postoperative period, visual acuity either improved or the improvement in vision which had been induced by pre-operative steroid treatment was maintained as the steroid dosage was reduced. However, on reviewing the long-term visual outcome, it became apparent that the patients could be subdivided into three groups (Fig. 2):

Group I. In 19 patients (58\%), visual acuity improved and remained stable following surgery. This visual improvement was maintained without the requirement for additional treatment once the postoperative steroids were discontinued.

Group II. In 12 patients (36\%), after an initial

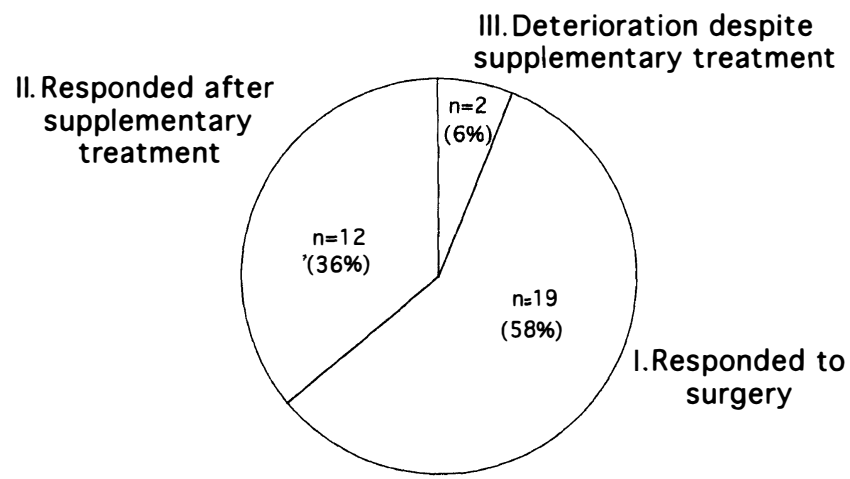

Fig. 2. Long-term response to orbital decompression in 33 patients. post-operative improvement in vision, visual acuity subsequently deteriorated. This responded to supplementary treatment (Fig. 3). In 4 patients, vision improved after a course of high-dose prednisolone. When the steroids were discontinued, visual acuity remained stable in 3 patients. The remaining patient subsequently relapsed and required a further course of steroids before visual function stabilised. In these patients visual deterioration occurred at a mean of 10 months post-operatively. Four patients required a course of steroids combined with further orbital decompression. In 3 cases, the further decompression was performed within 6 months of the initial surgery. Visual acuity stabilised following the additional surgery in all these cases. Two patients were treated with both steroids and radiotherapy. Radiotherapy was used as optic disc swelling persisted despite steroid treatment. Following radiotherapy the disc swelling resolved and visual acuity improved. Two patients were treated with radiotherapy alone postoperatively. Both patients had developed a slight deterioration in visual acuity combined with progressive visual field constriction. Following radiotherapy both visual acuity and visual fields improved. The patients who required radiotherapy received treatment between 6 and 16 months post-operatively.

Group III. In 2 patients, despite an initially satisfactory response to surgery, vision subsequently deteriorated and did not improve despite treatment with high-dose steroids and radiotherapy.

Optic disc swelling resolved in all but 1 case and pupillary defects resolved in all cases.

There was no relationship between the preoperative dose or duration of systemic prednisolone and the long-term visual outcome. Subsequent visual deterioration occurred in 9 of the 18 patients who were taking more than $20 \mathrm{mg}$ prednisolone daily at the time of surgery. This responded to supplementary treatment with either systemic steroids or radiotherapy.

The long-term post-operative visual acuities following decompression of the 65 orbits are

Radiotherapy alone

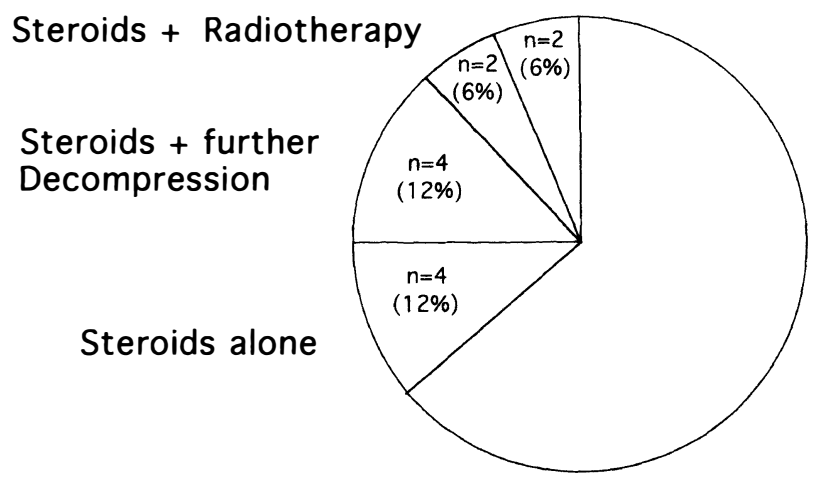

Fig. 3. Proportion of patients responding to supplementary treatment following orbital decompression. 


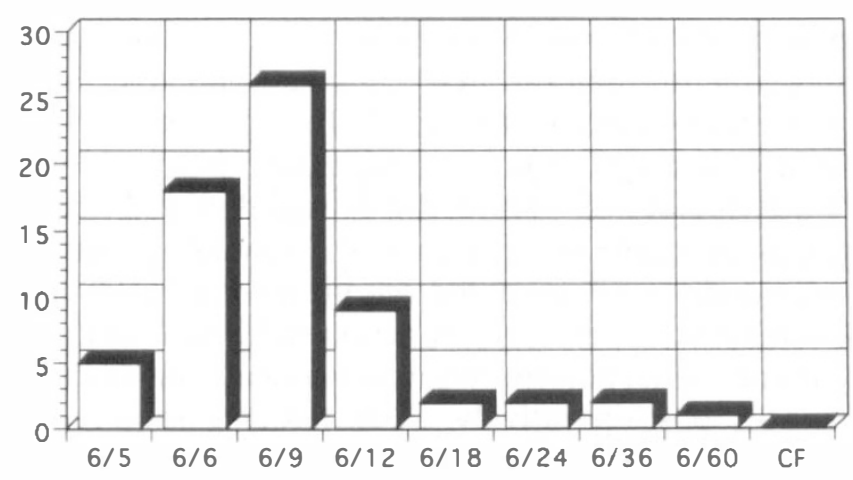

Fig. 4. Long-term post-operative visual acuity ( $\mathrm{n}=65$ eyes; mean follow-up 46 months).

summarised in Fig. 4. The scattergraph in Fig. 5 compares the presenting visual acuities with the current level of vision.

The relationship between length of follow-up and final visual outcome indicated that visual function stabilised within 12 months of surgery in $76 \%$, and within 18 months in $91 \%$ of patients. One patient's vision deteriorated $4 \frac{1}{2}$ years after an initially good response to surgery.

The most common complication was a sensation of numbness or tingling in the distribution of the infraorbital nerve, although the nerve had been identified and spared at the time of surgery in all cases. All patients experienced this initially and whereas symptoms did improve with time, they did not resolve completely, and remained troublesome in several patients. ${ }^{8}$

Two patients without pre-operative diplopia subsequently developed diplopia which required surgery. Five patients who had pre-operative diplopia also went on to require strabismus correction.

One patient developed transient bilateral failure of adduction immediately post-operatively. The probable mechanism for this unusual complication was compression of the third cranial nerve supply to the medial recti, owing to these nerves occupying an anatomically abnormal position. ${ }^{9}$

There were no cases of visual loss or sinusitis following surgery.

\section{DISCUSSION}

Compressive optic neuropathy is a potentially blinding complication of thyroid eye disease which requires urgent treatment. Medical treatment with high-dose systemic corticosteroids is effective in many patients. ${ }^{10}$ However, relapses often occur when the dosage is reduced, ${ }^{11,12}$ and the systemic side effects associated with the required dose limit their use in the long term. Orbital radiotherapy has also been shown to be a useful treatment for compressive optic neuropathy. The reported relapse rates following this method of treatment have been variable. ${ }^{11,13,14}$ Surgical orbital decompression has an

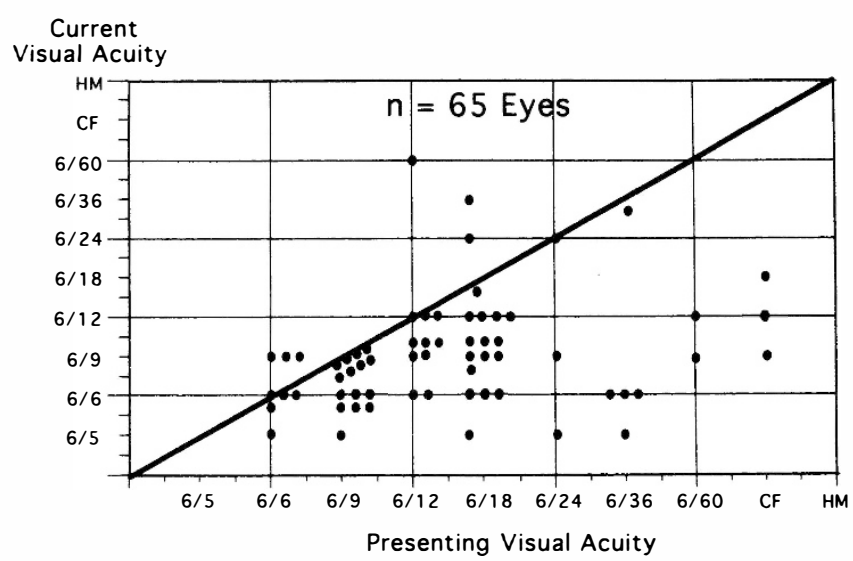

Fig. 5. Scatterplot comparing presenting and current visual acuities.

immediate effect by relieving compression of the optic nerve at the orbital apex. The beneficial effects of this treatment on visual function are well described. ${ }^{2-7}$

Our results are encouraging in that visual improvement was achieved in the short term and maintained in the long term in the majority of our patients (Figs. 4, 5). Fifty-eight per cent required no further treatment and an additional $36 \%$ had a transient deterioration which responded to supplementary treatment. These results compare favourably with the results of other authors. Hallin et al. ${ }^{4}$ found that the vision in $77 \%$ of their patients improved after orbital decompression. Warren et $a l .{ }^{5}$ commented that visual acuity improved or was maintained at the pre-operative level in $95 \%$ of their patients. In the 453 eyes with a visual acuity of less than 20/20 described by Garrity et al., ${ }^{6} 89 \%$ improved or remained the same following decompression.

We did not find worsening of extraocular muscle balance to be a significant post-operative complication. Only $6 \%$ of our patients developed diplopia, which subsequently required surgical correction, as a new event following decompression. $\mathrm{McCord}^{7}$ highlighted the relatively low incidence of post-operative diplopia associated with the translid, rather than the transantral, approach. In his series he found a 5.6\% incidence of worsened muscle balance following the translid approach in contrast to the $40 \%$ worsening of muscle balance following the transantral method.

In this series, surgical orbital decompression for compressive optic neuropathy seems to result in a good long-term visual outcome. However, there remains a small group of patients $(6 \%)$ in whom the visual function continued to deteriorate despite all forms of treatment. Perhaps this serves to highlight the fact that surgery merely alters the volume-to-space discrepancy within the orbit and has no effect on the underlying cause of this unpredictable condition. 
Key words: Dysthyroid optic neuropathy, Graves' disease, Orbital decompression.

\section{REFERENCES}

1. Char DH. Thyroid eye disease. Baltimore: Williams and Wilkins, 1985.

2. Calcaterra TC, Thompson JW. Antral-ethmoidal decompression of the orbit: ten year experience. Laryngoscope 1980;90:1941-9.

3. DeSanto LW. The total rehabilitation of Graves' ophthalmopathy. Laryngoscope 1980;90:1652-78.

4. Hallin ES, Feldon SE, Luttrell J. Graves' ophthalmopathy. III. Effect of transantral orbital decompression on optic neuropathy. Br J Ophthalmol 1988;72:683-7.

5. Warren JD, Spector JG, Burde R. Long-term follow-up and recent observations on 305 cases of orbital decompression for dysthyroid ophthalmopathy. Laryngoscope 1989;99:35-40.

6. Garrity JA, et al. Results of transantral orbital decompression in 428 patients with severe Graves' ophthalmopathy. Am J Ophthalmol 1993;116:533-47.
7. McCord CD. Current trends in orbital decompression. Ophthalmology 1985;92:21-33.

8. Hutchison BM, Kyle PM. Infraorbital nerve recovery following orbital decompression. Orbit 1991;10:41-4.

9. Kinsella F, Kyle PM, Stansfield A. Bilateral failure of adduction following orbital decompression. $\mathrm{Br} \mathrm{J}$ Ophthalmol 1990;74:239-41.

10. Day RM, Carroll FD. Corticosteroids in the treatment of optic nerve involvement associated with thyroid dysfunction. Arch Ophthalmol 1968;79:279-82.

11. Kazim M, Trockel S, Moore S. Treatment of acute Graves' orbitopathy. Ophthalmology 1991;98:1443-8.

12. Leone CR. The management of ophthalmic Graves' disease. Ophthalmology 1984;91:770-9.

13. Panzo GJ, Tomsak RL. A retrospective review of 26 cases of dysthyroid optic neuropathy. Am J Ophthalmol 1983;96:190-4.

14. Hurbli $\mathrm{T}$, et al. Radiation therapy for thyroid eye disease. Am J Ophthalmol 1985;99:633-7. 